\title{
DETECTION OF PERSISTING PHOTOELECTRONS IN AlGaAs DOUBLE HETEROSTRUCTURE LASER DIODES BY DLTS*
}

\author{
S. BREHME \\ Central Institute of Electron Physics, Academy of Science of GDR, \\ Hausvogteiplatz 5-7, Berlin 1086, Germany \\ (Received August 8, 1990) \\ LPE-made AlGaAs double heterostructure laser diodes having a Sn-doped \\ $n$-type confinement layer were investigated. A significant change of the low- \\ -temperature part of DLTS spectra and $C(T)$ curves was observed after \\ applying forward or higher reverse voltage. Relaxation of the curves took \\ several hours. This persistent photoconductivity phenomenon is explained \\ by photoionization of the DX centres.
}

PACS numbers: 71.55.Eq

\section{Introduction}

$\mathrm{Al}_{x} \mathrm{Ga}_{1-x} \mathrm{As}$ reveals the presence of dopant-related deep electron traps for $x>0.2$ partly in a concentration higher than the concentration of shallow donors. These so-called DX levels [1] show peculiar features such as coupling of emission and capture processes to higher bands direct material $(x<0.43)$ and strongly thermally activated capture behaviour [2]. The latter property leads to persistent photoconductivity (PPC) at lower temperatures since the recapture of photoemitted electrons by empty traps takes several hours [3].

\section{Samples}

Double heterostructure laser diodes (DH LD) were made by liquid phase epitaxy (LPE). The layers have been the following ones: $n$-type GaAs:Si (substrate, $100 \mu \mathrm{m}$ ), $n$-type GaAs:Si (buffer layer), $n$-type $\mathrm{Al}_{0.35} \mathrm{Ga}_{0.45} \mathrm{As}: \mathrm{Sn}$ (cladding layer, $2 \mu \mathrm{m}$ ), $p$-type $\mathrm{Al}_{0.05} \mathrm{Ga}_{0.95} \mathrm{As}$, partly $\mathrm{Ge}$-doped (active layer, $0.15 \mu \mathrm{m}$ ), $p$-type $\mathrm{Al}_{0.35} \mathrm{Ga}_{0.65} \mathrm{As}: \mathrm{Mg}$, Ge (cladding layer, $2 \mu \mathrm{m}$ ), $p$-type $\mathrm{GaAs}$ :Ge (contact layer).

*This work was supported in part by Werk für Fernsehelektronik Berlin. 


\section{Results}

In the DLTS spectra two Sn-related DX centres called DX1 and DX2 here were always found independently of the $p$-side dopant (Fig. 1, spectrum (a)). The localization of DX1, DX2 in the $n$-type cladding layer was confirmed by profile measurements (DDLTS).

$E_{\mathrm{t}, 2}=0.33 \pm 0.03 \mathrm{eV}$ was found for DX2. The DX1 should be the main Sn-related DX centre $\left(E_{\mathrm{t}, 1}=0.19 \ldots 0.21 \mathrm{eV}\right.$, e.g. [4]). The low-temperature part of spectrum (a) was recorded under non-saturation conditions.

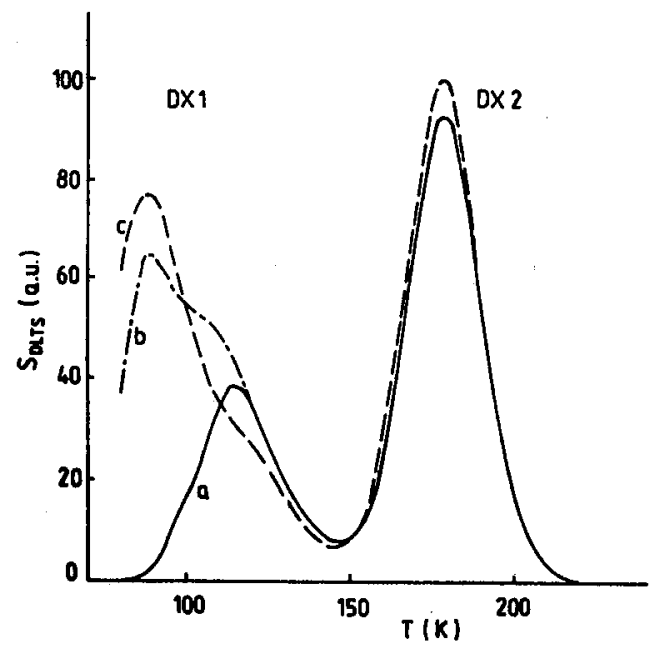

Fig. 1. DLTS spectra recorded under heating conditions. The rate window was $1180 \mathrm{~s}^{-1}$, the bias was $-5 \mathrm{~V}$. Spectrum (a) $(---)$, pulse duration $t_{\mathrm{p}}=100 \mu \mathrm{s}$. Spectrum (b) (- - ), as (a), but after a current pulse at $T_{\mathrm{LN}}$. Spectrum (c) (- - ), the first pulse was a current pulse $(10 \mu \mathrm{s})$. After $5 \mu$ s delay a majority carrier pulse as in (a), (b) was applied $(90 \mu \mathrm{s})$.

The DX1-related signal was strongly increased in spcctra (b) (as (a), but after current injection at $T_{\mathrm{LN}}$ ) and (c) (double pulse excitation: the first pulse current injection, the second pulse - majority carrier pulse). Similar results were obtained after applying higher reverse bias $(-9 \ldots-14 \mathrm{~V})$ to the samples for some minutes instead of forward bias.

The relaxation of the sample capacity $C_{\circ}$ and of the DLTS signal at $T_{\mathrm{LN}}$ after current injection is shown in Fig. 3. In the first $10 \mathrm{~s}$ not shown here there were a quick and strong decay of $C_{\mathrm{o}}$ (comp. [5]) which corresponds to the difference between records $\mathrm{C}$ and $\mathrm{D}$ in Fig. 2. 


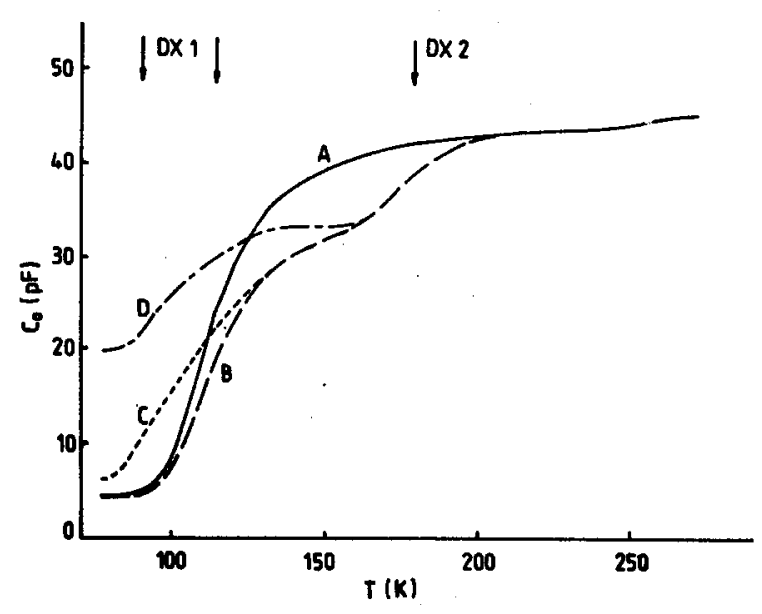

Fig. 2. $\quad C-T$ records at a bias of -5 V. Tracks $\mathrm{B}(-), \mathrm{C}(----)$ and $\mathrm{D}(---)$ were records during the DLTS measurements of Fig. 1: tracks $B, C, D$ correspond to spectra (a), (b), (c), respectively. Arrows indicate the positions of the DLTS peaks.

\section{Interpretation}

The increase of the DX1-related DLTS signal after current injection is caused by a better electron filling degree of the trap at the end of the excitation pulse:

$$
f_{n}=1-\exp \left(-c_{n} t_{p}\right)
$$

with

$$
c_{n}=n \sigma_{n} \bar{v}_{n}
$$

and $n$ - the free electron concentration, $\sigma_{n}$ - the electron capture cross section, $\bar{v}_{n}$ - the mean thermal electron velocity.

The concentration of free electrons in the $n$-type cladding layer is rather low at $T_{\mathrm{LN}}$ due to freezing effects. During the current flow through the DH LD light is generated $(h \nu \approx 1.5 \mathrm{eV})$ which is well able to photoionize the DX centres $[6,7]$. Because of the PPC effect a strong increase of the free electron concentration is caused.

The persisting photoelectrons also reduce the serial resistance contribution from the $n$-type cladding layer as observed in the $I-V$ characteristics for forward bias at $T_{\mathrm{LN}}$.

The cause of the increased free electron density after applying higher reverse bias could be avalanche process-related light emission. It is well known that avalanche processes in GaAs can produce light emission phenomena, e.g. [8].

The slow component of the decay curves (Fig. 3 ) is related to DX2, the quick initial decay to the main trap DX1. This is supported by photoconductivity and DLTS measurements [7] and by capture data reported, e.g. [4]. That means the 


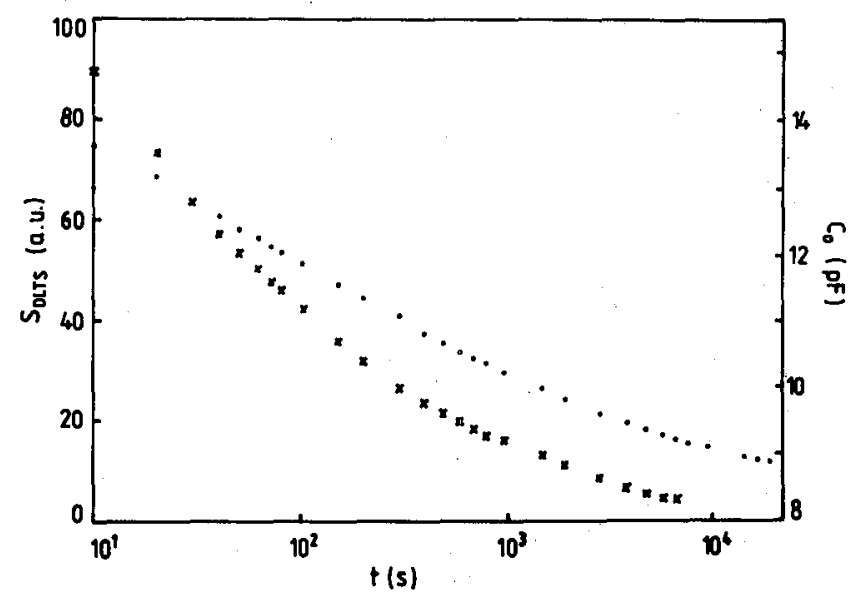

Fig. 3. Decay of the DLTS signal $(\bullet$, left ordinate) and of the sample capacity ( $\times$, right ordinate) at $T_{\mathrm{LN}}$ after a current pulse. The conditions of the DLTS measurement were: rate window $11.8 \mathrm{~s}^{-1}$, pulse length $10 \mathrm{~ms}$, bias $-5 \mathrm{~V}$, pulse height $5 \mathrm{~V}$.

PPC effect in $n$-type $\mathrm{Al}_{0.35} \mathrm{Ga}_{0.65} \mathrm{As}: \mathrm{Sn}$ at and above $T_{\mathrm{LN}}$ is mainly related to DX2 and not to the dominant trap DX1.

\section{References}

[1] D.V. Lang, R.A. Logan, M. Jaros, Phys. Rev. B 19, 1015 (1979).

[2] P.M. Mooney, Material Res. Soc. Symp. Proc. 104, 561 (1988).

[3] R.J. Nelson, Appl. Phys. Lett. 31, 351 (1977).

[4] B. Balland, J.L. Pavot, B.B. de Cremoux, P. Hirtz, Phys. Status Solidi A 68, 661 (1981).

[5] K. Zdansky, L.T. Binh, Phys. Status Solidi A 116, 357 (1989).

[6] D.V. Lang, in Deep Centers in Semiconduktors, ed. S.T. Pantelides, Gordon and Breach Science Publishers, New York 1986, p. 489.

[7] D. Huber, personal communication.

[8] J. Chen, B. Gao, D. IIuang, J.I. Chyi, M.S. Ünlü, II. Morkoç, Appl. Phys. Lett. 55, 374 (1989). 